\title{
Conservation of Queen Pheromones Across Two Species of Vespine Wasps
}

\author{
Cintia A. Oi ${ }^{1}$ • Jocelyn G. Millar ${ }^{2}$ • Jelle S. van Zweden ${ }^{1} \cdot$ Tom Wenseleers $^{1}$
}

Received: 29 April 2016 /Revised: 21 August 2016/Accepted: 19 September 2016

(C) Springer Science+Business Media New York 2016

\begin{abstract}
Social insects are known for their reproductive division of labor between queens and workers, whereby queens lay the majority of the colony's eggs, and workers engage mostly in non-reproductive tasks. Queens produce pheromones that signal their presence and fertility to workers, which in turn generally remain sterile. Recently, it has been discovered that specific queen-characteristic cuticular hydrocarbons (CHCs) function as queen pheromones across multiple lineages of social insects. In the common wasp, Vespula vulgaris, several long-chain linear alkanes and 3-methylalkanes were shown to act as queen signals. Here, we describe similar bioassays with a related species of highly eusocial vespine wasp, the Saxon wasp, Dolichovespula saxonica. We show that a blend of queencharacteristic hydrocarbons that are structurally related to those of the common wasp inhibit worker reproduction, suggesting conservation of queen pheromones across social wasps. Overall, our results highlight the central importance of CHCs in chemical communication among social insects in general, and as conserved queen pheromones in these social wasps in particular.
\end{abstract}

Keywords Queen pheromone · Cuticular hydrocarbon · Social insect · Dolichovespula saxonica, Vespula vulgaris

Electronic supplementary material The online version of this article (doi:10.1007/s10886-016-0777-9) contains supplementary material, which is available to authorized users.

Cintia A. Oi

cintiaakemioi@gmail.com

1 Laboratory of Socioecology and Social Evolution, KU Leuven University of Leuven, Naamsestraat 59, 3000 Leuven, Belgium

2 Departments of Entomology and Chemistry, University of California, Riverside, CA 92521, USA

\section{Introduction}

Social insect colonies are well known for their marked reproductive division of labor between queens and workers, in which queens produce the majority of the colony's eggs, and workers engage mostly in non-reproductive tasks. This reproductive division of labor is regulated by the queen through the production of queen pheromones, which signal the queen's presence and fertility, and cause most workers to refrain from reproducing (Holman et al. 2010, 2013; Le Conte and Hefetz 2008; Oi et al. 2015a; Van Oystaeyen et al. 2014). In contrast to the honeybee Apis mellifera, in which much work has focused on the queen's mandibular pheromones and their major constituent 9-oxo-(E)-2-decenoic acid (9-ODA), it has been shown that cuticular hydrocarbons (CHCs) likely act as conserved queen signals across several other lineages of social Hymenoptera (Holman et al. 2013; Oi et al. 2015a; Oliveira et al. 2015; Van Oystaeyen et al. 2014). In particular, bioassays have shown that specific queen-characteristic hydrocarbons inhibit worker reproduction across a variety of social insect taxa, including the bumblebee Bombus terrestris (Holman 2014; Van Oystaeyen et al. 2014), the common wasp Vespula vulgaris (Van Oystaeyen et al. 2014), and several species of ants (Holman et al. 2010, 2013, 2016a; Smith et al. 2016; Van Oystaeyen et al. 2014). These results demonstrate that specific CHCs have evolved to become queen pheromones across at least three independent origins of eusociality (Oi et al. 2015a; Oliveira et al. 2015; Van Oystaeyen et al. 2014). Comparative analyses further showed that hydrocarbons are the most common class of compounds that appear as queen-characteristic signals in social Hymenoptera (Holman et al. 2013; Oliveira et al. 2015; Van Oystaeyen et al. 2014). It is likely that the convergent evolution of hydrocarbon queen pheromones is driven by the fact that specific CHCs correlate with reproductive state in both social and non-social insect species (Blomquist and Bagnères 
2010; Liebig 2010; Oi et al. 2015a), which predisposes them to be co-opted as honest signals of fertility (Holman 2012; Monnin 2006; van Zweden 2010).

The studies cited above suggest that social insect queen pheromones show strong evolutionary conservation, even though this conclusion was based mostly on comparison of distantly related clades, and hence could be due to convergent evolution. Only a limited number of studies have tested for conservation of these signals within closely related species. Data from Apis bees (Plettner et al. 1997), Temnothorax ants (Brunner et al. 2011), and Lasius ants (Holman et al. 2013, 2016a) suggest that queen pheromones have remained relatively stable over evolutionary time. Some studies even have shown that queen pheromones can function interspecifically to inhibit worker reproduction (Brunner et al. 2011; Holman et al. 2013). On the other hand, there are examples where fertility signals do not appear to be conserved within a particular genus. Most notably, in Odontomachus trap-jaw ants, a comparison across three congeneric species showed that fertility signals were not conserved, with chemical compounds from queens and workers differing in number and type among the species analyzed (Smith et al. 2016). It also was shown that the chemical context in which the signal is perceived can be important for proper recognition of fertility signals (Smith et al. 2015). In addition, in the buff-tailed bumblebee, Bombus terrestris, the queen-characteristic linear alkane pentacosane induced ovary regression (Van Oystaeyen et al. 2014), whereas in the related species $B$. impatiens, pentacosane was claimed to have no activity as a queen pheromone (Amsalem et al. 2015). However, reanalysis of Amsalem et al.'s data with more powerful statistical models did indeed indicate that pentacosane reduced and delayed egg-laying by workers, thereby challenging the suggested lack of conservation (Holman et al. 2016b).

Here, as a further test of possible conservation of these signals, we describe bioassays with a related species of the highly eusocial vespine wasps: the Saxon wasp, Dolichovespula saxonica. The typical queen-characteristic CHCs of this species are linear and 3-methylalkanes, and thus structurally related to the queen pheromones of the common wasp, Vespula vulgaris (van Zweden et al. 2014). We therefore hypothesized that a synthetic blend of these queencharacteristic hydrocarbons would inhibit worker ovary development, and we found that this indeed was the case, supporting the hypothesis that queen pheromones are highly conserved among social wasps.

\section{Methods and Materials}

Shortlisting of Potential Queen Pheromone Components To identify possible queen pheromone components, we collected three natural colonies of Dolichovespula saxonica in the vicinity of Leuven, Belgium. Cuticular hydrocarbons were extracted by immersing the wasps (10 workers per colony and each of the mother queens) in $1.5 \mathrm{ml}$ pentane (HPLC grade, Sigma-Aldrich). The pentane was evaporated, the extract reconstituted in $250 \mu \mathrm{l}$ hexane, and $2 \mu \mathrm{l}$ injected in a Shimadzu QP2010 Ultra GC-MS (gas chromatograph-mass spectrometer). Injections were made in split mode (10:1), using an injection temperature of $280{ }^{\circ} \mathrm{C}$, a DB-5 MS capillary column $(30 \mathrm{~m} \times 0.25 \mathrm{~mm} \times 0.25 \mu \mathrm{m}$ film thickness; J \& W Scientific, Folsom CA, USA), and helium as the carrier gas with a flow rate of $1 \mathrm{ml} / \mathrm{min}$. The oven temperature was held at $70{ }^{\circ} \mathrm{C}$ for $1 \mathrm{~min}$, then increased to $220^{\circ} \mathrm{C}$ at $25{ }^{\circ} \mathrm{C} / \mathrm{min}$, and then to $325{ }^{\circ} \mathrm{C}$ at $3{ }^{\circ} \mathrm{C} / \mathrm{min}$, with a final hold of $15 \mathrm{~min}$ at $325^{\circ} \mathrm{C}$. Peak areas of $65 \mathrm{CHCs}$ were integrated using a custom script in R version 3.1.0 (Team RC 2012) (available from the authors upon request). The software AMDIS 2.71 was used in combination with NIST MS Search 2.2 and the NIST 2014 mass spectral database search and manual interpretation of mass spectra (taking into account expected fragmentation patterns and retention indices) to identify the different compounds. Alkene double bond positions were tentatively identified on the basis of measured retention indices of known alkene standards that were run on our machine on the same temperature program and whose double bond positions were checked using dimethyldisulfide (DMDS) derivatization (Carlson et al. 1989). Subsequently, we performed a principal components analysis (PCA) on Z-transformed relative peak areas (Aitchison 1986) to look at patterns of variation within our dataset, using the software packages FactoMineR (Lê et al. 2008) and psych (Revelle 2016). In addition, to short list compounds to be tested in bioassays, we fitted separate univariate linear mixed models on $\log _{2}$ transformed relative peak areas for all 65 compounds present (Table S1). In these analyses, colony and caste were coded as random and fixed factors, respectively. Compounds then were ranked according to how queen characteristic they were, which was done on the basis of the mean fold difference in abundance on the cuticle of queens $v s$. workers (Table S2). This analysis revealed that the linear alkanes nonacosane $\left(n-\mathrm{C}_{29}\right)$, triacontane $\left(n-\mathrm{C}_{30}\right)$, and hentriacontane $\left(n-\mathrm{C}_{31}\right)$, and the 3-methylalkanes 3-methylnonacosane (3- $\mathrm{MeC}_{29}$ ) and 3-methylhentriacontane (3- $\left.\mathrm{MeC}_{31}\right)$ were among the most queen-characteristic compounds, as well as structurally similar to the queen pheromones of $\mathrm{V}$. vulgaris (heptacosane $n$ - $\mathrm{C}_{27}$, nonacosane $n-\mathrm{C}_{29}$, and 3-methylnonacosane 3- $\mathrm{MeC}_{29}$ ) (Van Oystaeyen et al. 2014). The queen-characteristic alkene 7hentriacontene $\left(7-\mathrm{C}_{31: 1}\right)$ and dimethylalkane 3,7dimethylnonacosane $\left(3,7-\mathrm{diMeC}_{29}\right)$ were not tested because they were not available to us.

Bioassays For the bioassays, we collected five $D$. saxonica colonies in the vicinity of Leuven and transferred them to closed wooden boxes $(36 \times 15 \times 15 \mathrm{~cm})$ held at approximately $28^{\circ} \mathrm{C}$, where they were fed sugar paste (Apifonda) and Tenebrio 
mealworms ad libitum [similar to Oi et al. (2015b)]. At the time of collection, all colonies contained a healthy queen. At the start of our experiment, the queens were removed so that we could test if the queen-characteristic $\mathrm{CHCs}$ inhibited worker ovary development analogous to a natural queen. Because the nests were small (ca. 75 workers each), they were split into 2 approximately equal parts, of which one served as control and the other as the queen-pheromone treated group. Treatment were applied daily by pipetting $150 \mu \mathrm{l}$ of either pentane for the control or a pentane solution of a blend of the queen-characteristic hydrocarbons, at a concentration of two queen equivalents/day (for $n-\mathrm{C}_{29} 54 \mu \mathrm{g}, 3-\mathrm{MeC}_{29} 25.5 \mu \mathrm{g}, n-\mathrm{C}_{30} 3.5 \mu \mathrm{g}, n-\mathrm{C}_{31} 8.1 \mu \mathrm{g}$, $3-\mathrm{MeC}_{31} 5.3 \mu \mathrm{g}$ ), on top of the comb for $2 \mathrm{wk}$. Absolute concentrations were quantified by using an external alkane ladder ( $n-\mathrm{C}_{7}$ to $n-\mathrm{C}_{40}$, Supelco, Bellefonte PA, USA). Linear alkanes were obtained from Sigma-Aldrich, and the methylalkanes were synthesized using the procedures described in Van Oystaeyen et al. (2014). Subsequently, all workers were frozen at $-20{ }^{\circ} \mathrm{C}$ for later dissection. To minimize observer bias, all dissections were carried out blind with respect to treatment. As in previous studies (van Zweden et al. 2014; Wenseleers and Ratnieks 2006), ovaries were considered activated when the largest oocyte was larger than half the size of a freshly-laid egg.

Proportions of workers with active ovaries were compared between each treatment and control using a generalized linear mixed model (GLMM) with binomial errors and logit link function, whereby colony was coded as a random intercept. Colony size was not significant, therefore was not included in the model. The chosen model had the highest explanatory power as measured by a low AIC (Aikaike Information Criterion). These analyses were performed using the package lme4 in R v3.1.0 (Team RC 2012). The hydrocarbon compounds were tested only as a blend and not individually because splitting colonies into more than two treatment groups was not possible due to their small size in this study species (ca. 50-100 workers in the growth phase). In addition, an unpaired experimental design using different colonies across the different treatment groups was deemed inadvisable because this could have resulted in confounding effects linked to variation in colony size and development phase, sociogenetic structure, and genetic background, which have been suggested to have large effects on levels of worker reproduction in this species (Bonckaert et al. 2011; Foster and Ratnieks 2000).

\section{Results}

We identified 61 hydrocarbons in the CHCs of queens and workers (Fig. 1; Table S1). A principal components analysis (PCA) showed a clear separation between queens and workers, and revealed that a similar set of hydrocarbons was characteristic of queens as in a previously studied Danish population, including the alkanes $n-\mathrm{C}_{29}, 3-\mathrm{MeC}_{29}, 3,7-$ diMeC $29, n-\mathrm{C}_{31}$, and $3-\mathrm{MeC}_{31}$ and the alkene $7-\mathrm{C}_{31: 1}$ (Fig. 1) (van Zweden et al. 2014). The same compounds also emerged as characteristic for queens based on univariate tests (Table S2). Bioassays revealed that application of a blend of the queen-characteristic $\mathrm{CHCs} n-\mathrm{C}_{29}, 3-\mathrm{MeC}_{29}, n-\mathrm{C}_{30}, n-\mathrm{C}_{31}$, and $3-\mathrm{MeC}_{31}$ indeed resulted in a significant (ca. 2 fold) reduction in the odds for workers to activate their ovaries compared to the solvent-only control (Fig. 2, Table S3; total of $N=223$ and 153 dissected workers in the treatment and control conditions, respectively; $12.0 \%$ vs. $20.6 \%$; binomial GLMM, coefficient $=-0.65$, odds ratio $=0.52, z=-2.07$, $P=0.038$ ). This result demonstrates that one or more compounds in the blend were bioactive queen pheromones.

\section{Discussion}

We showed that application of a blend of the queencharacteristic hydrocarbons $n-\mathrm{C}_{29}, 3-\mathrm{MeC}_{29}, n-\mathrm{C}_{30}, n-\mathrm{C}_{31}$, and $3-\mathrm{MeC}_{31}$ reduces activation of worker ovaries in the Saxon wasp, Dolichovespula saxonica, thereby demonstrating that one or more of these compounds acts as a queen pheromone to mediate reproductive division of labor in this species. Two of these compounds, $n-\mathrm{C}_{29}$ and $3-\mathrm{MeC}_{29}$, have been shown to be queen pheromone components in the common wasp Vespula vulgaris (Van Oystaeyen et al. 2014). In addition, the queen-characteristic CHCs identified in this study were almost the same as those reported from a Danish population of the same species (van Zweden et al. 2014). These results, therefore, suggest conservation of queen pheromones in social wasps among different populations as well as across related species.

The conservation of these signals in social wasps supports the "honest signal" hypothesis for the evolution of social insect queen pheromones (Holman 2012; Keller and Nonacs 1993; Monnin 2006; Oi et al. 2015a; Peso et al. 2015; van Zweden 2010). This hypothesis proposes that workers should be selected to respond to queen pheromones only to the extent that it serves their own inclusive fitness interests (Keller and Nonacs 1993). Under this hypothesis, the alignment of the interests of the queen and workers would be predicted to lead to slow and gradual evolution of queen signals (Heinze and d'Ettorre 2009). By contrast, the alternative hypothesis that the queen uses pheromones to manipulate workers to remain sterile against their own interests ("queen control") predicts that queen pheromones should evolve quickly due to the coercive rather than cooperative nature of such a relationship (Kocher and Grozinger 2011; Oi et al. 2015a). Thus far, experimental data from honeybees and Temnothorax and Lasius ants have demonstrated relatively strong conservation of queen pheromones (Brunner et al. 2011; Holman et al. 2013; Keller and Nonacs 1993; Oldroyd and Wongsiri 2006). Phylogenetic 
Fig. 1 a Cuticular hydrocarbons (CHC) profiles of queens (top, purple) and workers (bottom, blue) of Dolichovespula saxonica, as determined by GC-MS analysis. Dark purple arrows indicate compounds tested in our bioassays: $n-\mathrm{C}_{29}, 3-\mathrm{MeC}_{29}$, $n-\mathrm{C}_{30}, n-\mathrm{C}_{31}$, and $3-\mathrm{MeC}_{31}$. Grey dotted arrows indicate compounds not tested, but abundant in queens: 3,7-diMeC 29 and $7-\mathrm{C}_{31: 1}$ b Principal components analysis (PCA) of CHC profiles ( $N=3$ colonies) shows separation between queens and workers (left panel) and the squares indicate the centroids of both castes (queens in purple and workers in blue) and colonies (in grey). The right panel shows the factor loadings. Five compounds shown in dark purple were used as the treatment blend in our bioassays (Online version in color)
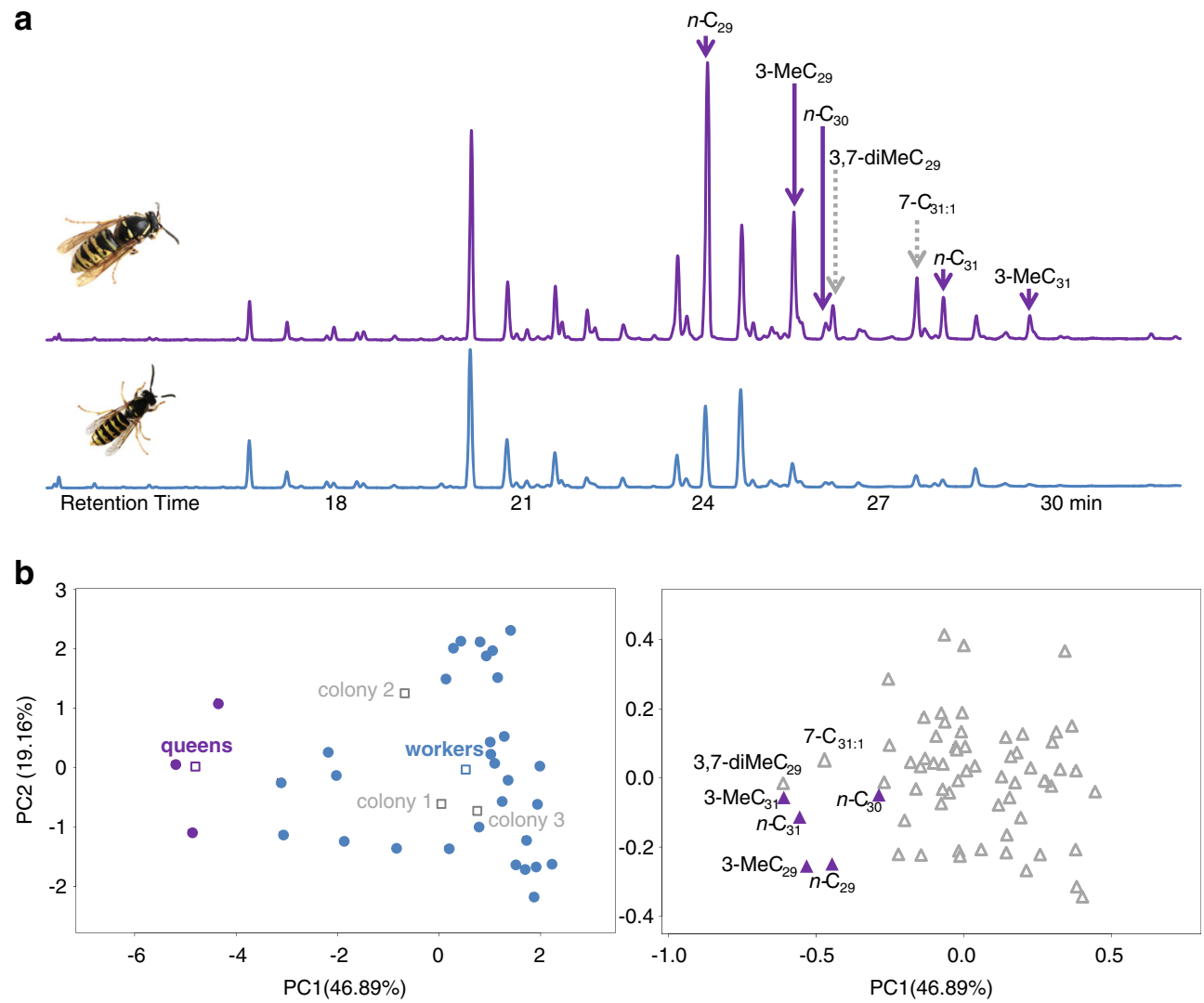

analyses of queen- and fertility-linked compounds in social Hymenoptera (Van Oystaeyen et al. 2014) and corbiculate bees (Oliveira et al. 2015) suggest that queen pheromones evolve slowly, thus supporting the honest signalling hypothesis [but see Smith et al. (2016)], for an example in Odontomachus ants where fertility signals apparently are less conserved).

Our results contrast with the hypothesis that hydrocarbon profiles would be too complex and too variably expressed (Howard and Blomquist 2005) to act as reliable castespecific signals (Amsalem et al. 2015). Although we currently cannot determine if the $D$. saxonica worker response to queen pheromones was learned (e.g., in the form of habituation) (Amsalem et al. 2015; Wyatt 2014), all that appears to be required for workers to recognize the presence of a queen is to discriminate the relative amounts of a subset of the CHCs that are characteristic of the queen. Such discrimination does not seem unlikely, because both ants and bees are able to accurately discriminate among different hydrocarbons differing only in chain length or by the position of a methyl group, including those hydrocarbons used as fertility signals (Sharma et al. 2015) and nestmate recognition cues (Châline et al. 2005; di Mauro et al. 2015). Experimental evidence also has shown that specific hydrocarbon compounds can elicit reproducible and conserved responses as queen signals, in contrast to some of the other compounds that are present on the insects' cuticle (de Narbonne et al. 2016; Holman et al. 2010, 2016a, 2016b; Van Oystaeyen et al. 2014). In fact, the mechanism of specific hydrocarbons acting as queen pheromones may still work even if the response to queen pheromones depends partly on chemical context (Smith et al. 2016).

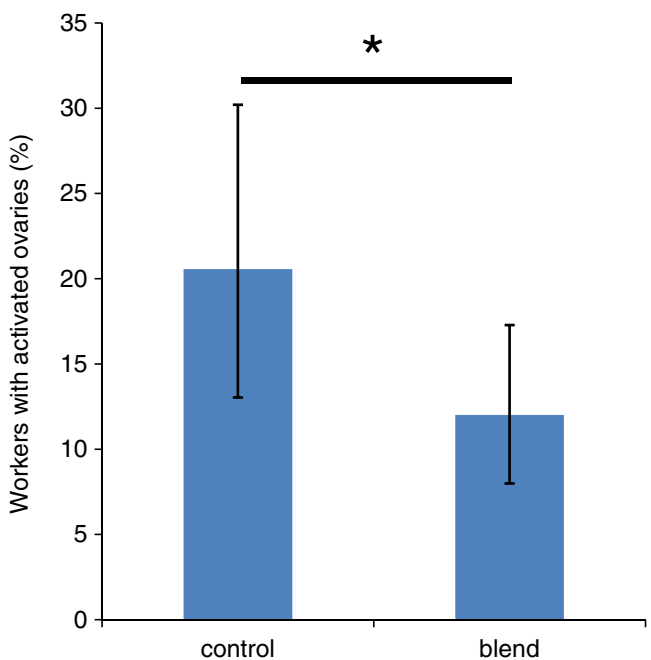

Fig. 2 Percentage of workers with activated ovaries after 2 wk. of daily treatment with a blend of the queen-characteristic cuticular hydrocarbons $n-\mathrm{C}_{29}, 3-\mathrm{MeC}_{29}, n-\mathrm{C}_{30}, n-\mathrm{C}_{31}$, and $3-\mathrm{MeC}_{31}$ dissolved in pentane, compared to a pentane-only control. Bars depict means $\pm 95 \%$ confidence limits as predicted from a fitted binomial mixed model. ( $N=5$ replicate colonies, $n=223$ and 153 dissected workers in treatment and control conditions, respectively, binomial GLMM, coefficient $=-0.64, z=-2.07, * P=0.03)$ (Online version in color) 
In summary, our study reinforces the hypothesis that specific CHCs play a central role in regulating the reproductive division of labor in social insects, and demonstrates that one or more of these compounds act as queen pheromones. This result is in agreement with predictions of the honest signalling hypothesis, thereby suggesting that specific CHCs may act as generic queen pheromones across vespine wasps. Further work is required to determine whether only one or a subset of the compounds tested here represents the bioactive queen pheromone. In addition, comparative studies, including the study of solitary outgroups, could reveal how hydrocarbon queen pheromones originated and from which ancestral signals or cues in solitary species they might be derived (Oi et al. 2015a; Van Oystaeyen et al. 2014).

Acknowledgments We thank H. Munks and A. Vandoren for help with field work. This study was supported by FWO (Research Fund - Flanders) grant G.0 A51.15 and KU Leuven Centre of Excellence grant PF/2010/007 (to T.W.), postdoctoral fellowship FWO 12Q7615N (to J.S.v.Z.), CNPq (Conselho Nacional de Desenvolvimento Científico e Tecnológico) Brazil scholarship 201959/ 2012-7 (to C.A.O.) and Hatch project CA-R*ENT-5181-H (to J.G.M.). The authors declare that they have no conflict of interest.

\section{References}

Aitchison J (1986) The statistical analysis of compositional data, vol 44. vol 2. Blackburn Press, Caldwell

Amsalem E, Orlova M, Grozinger CM (2015) A conserved class of queen pheromones? Re-evaluating the evidence in bumblebees (Bombus impatiens). Proc R Soc Lond B Biol Sci 282:20151800

Blomquist GJ, Bagnères AG (2010) Insect hydrocarbons: biology, biochemistry and chemical ecology. Cambridge University Press, Cambridge, UK

Bonckaert W, van Zweden JS, d'Ettorre P, Billen J, Wenseleers T (2011) Colony stage and not facultative policing explains pattern of worker reproduction in the Saxon wasp. Mol Ecol 20:3455-3468

Brunner E, Kroiss J, Trindl A, Heinze J (2011) Queen pheromones in Temnothorax ants: control or honest signal? BMC Evol Biol 11:55

Carlson DA, Roan CS, Yost RA, Hector J (1989) Dimethyl disulfide derivatives of long chain alkenes, alkadienes, and alkatrienes for gas chromatography/mass spectrometry. Anal Chem 61:1564-1571

Châline N, Sandoz JC, Martin SJ, Ratnieks FLW, Jones GR (2005) Learning and discrimination of individual cuticular hydrocarbons by honeybees (Apis mellifera). Chem Senses 30:327-335

de Narbonne MM, van Zweden JS, Bello JE, Wenseleers T, Millar JG, d'Ettorre P (2016) Biological activity of the enantiomers of 3methylhentriacontane, a queen pheromone of the ant Lasius niger. J Exp Biol 219:1632-1638

di Mauro G, Perez M, Lorenzi MC, Guerrieri FJ, Millar JG, d'Ettorre P (2015) Ants discriminate between different hydrocarbon concentrations. Front Ecol Evol 3:133

Foster K, Ratnieks F (2000) Social insects - facultative worker policing in a wasp. Nature 407:692-693

Heinze J, d'Ettorre P (2009) Honest and dishonest communication in social Hymenoptera. J Exp Biol 212:1775-1779

Holman L (2012) Costs and constraints conspire to produce honest signalling: insights from an ant queen pheromone. Evolution 66: 2094-2105
Holman L (2014) Bumblebee size polymorphism and worker response to queen pheromone. Peer J 2:e604

Holman L, Jørgensen CG, Nielsen J, d'Ettorre P (2010) Identification of an ant queen pheromone regulating worker sterility. Proc R Soc Lond B Biol Sci 277:3793-3800

Holman L, Lanfear R, d'Ettorre P (2013) The evolution of queen pheromones in the ant genus Lasius. J Evol Biol 26:1549-1558

Holman L, Hanley B, Millar JG (2016a) Highly specific responses to queen pheromone in three Lasius ant species. Behav Ecol Sociobiol 70:387-392

Holman L, van Zweden JS, Oliveira RC, Van Oystaeyen A, Wenseleers T (2016b) Conserved queen pheromones in bumblebees: A reply to Amsalem et al. PeerJ 4:e2003v2001 doi:10.7287/peerj.preprints.2003v2001

Howard RW, Blomquist GJ (2005) Ecological, behavioral, and biochemical aspects of insect hydrocarbons. Annu Rev Entomol 50:371-393

Keller L, Nonacs P (1993) The role of queen pheromones in social insects: queen control or queen signal? Anim Behav 45:787-794

Kocher SD, Grozinger CM (2011) Cooperation, conflict, and the evolution of queen pheromones. J Chem Ecol 37:1263-1275

Le Conte Y, Hefetz A (2008) Primer pheromones in social Hymenoptera. Annu Rev Entomol 53:523-542

Lê S, Josse J, Husson F (2008) FactoMineR: an R package for multivariate analysis. J Stat Softw 25:1-18

Liebig J (2010) Hydrocarbon profiles indicate fertility and dominance status in ant, bee, and wasp colonies. In: Insect hydrocarbons: biology, biochemistry, and chemical ecology. Cambridge University Press, pp 282-324

Monnin T (2006) Chemical recognition of reproductive status in social insects. Ann Zool Fenn 43:515-530

Oi CA, van Zweden JS, Oliveira RC, Van Oystaeyen A, Nascimento FS, Wenseleers T (2015a) The origin and evolution of social insect queen pheromones: novel hypotheses and outstanding problems. BioEssays 37:808-821

Oi CA, Van Oystaeyen A, Oliveira RC, Millar JG, Verstrepen KJ, van Zweden JS, Wenseleers T (2015b) Dual effect of wasp queen pheromone in regulating insect sociality. Curr Biol 25:1638-1640

Oldroyd BP, Wongsiri S (2006) Asian honey bees: biology, conservation, and human interactions. Harvard University Press, Cambridge, Massachusetts and London, England

Oliveira RC, Oi CA, do Nascimento MM, Vollet-Neto A, Alves DA, Campos MC, Nascimento F, Wenseleers T (2015) The origin and evolution of queen and fertility signals in corbiculate bees. BMC Evol Biol 15:254

Peso M, Elgar MA, Barron AB (2015) Pheromonal control: reconciling physiological mechanism with signalling theory. Biol Rev 90: $542-559$

Plettner E, Otis GW, Wimalaratne PDC, Winston ML, Slessor KN, Pankiw T, Punchihewa PWK (1997) Species- and castedetermined mandibular gland signals in honeybees (Apis). J Chem Ecol 23:363-377

Revelle W (2016) Psych: Procedures for personality and psychological research. Northwestern University, Evanston, Illinois, USA. https://CRAN.R-project.org/package=psychVersion=1.6.9

Sharma KR, Enzmann BL, Schmidt Y, Moore D, Jones GR, Parker J, Berger SL, Reinberg D, Zwiebel LJ, Breit B, Liebig J (2015) Cuticular hydrocarbon pheromones for social behavior and their coding in the ant antenna. Cell Rep 12:1261-1271

Smith AA, Millar JG, Suarez AV (2015) A social insect fertility signal is dependent on chemical context. Biol Lett 11:20140947

Smith AA, Millar JG, Suarez AV (2016) Comparative analysis of fertility signals and sex-specific cuticular chemical profiles of Odontomachus trap-jaw ants. J Exp Biol 219:419-430

Team RC (2012) R: a language and environment for statistical computing. R Foundation for Statistical Computing, Vienna, Austria, 2012. ISBN 3-900051-07-0 
Van Oystaeyen A, Oliveira RC, Holman L, van Zweden JS, Romero C, Oi CA, d'Ettorre P, Khalesi M, Billen J, Wäckers F, Millar JG, Wenseleers T (2014) Conserved class of queen pheromones stops social insect workers from reproducing. Science 287:287-290

van Zweden JS (2010) The evolution of honest queen pheromones in insect societies. Comm Int Biol 3:50-52 van Zweden JS, Bonckaert W, Wenseleers T, d'Ettorre P (2014) Queen signalling in social wasps. Evolution 68:976-986

Wenseleers T, Ratnieks FL (2006) Enforced altruism in insect societies. Nature 444:50

Wyatt TD (2014) Pheromones and animal behavior: chemical signals and signature mixes. Cambridge University Press, Cambridge, UK 